\title{
Embedded surfaces in four-manifolds, branched covers, and $\mathrm{SO}(3)$-invariants
}

\author{
By D. KOTSCHICK \\ Mathematisches Institut, Universität Basel, Rheinsprung 21, 4051 Basel, Switzerland \\ AND G. MATIĆ* \\ Department of Mathematics, The University of Georgia, Athens, GA 30602, U.S.A.
}

(Received 23 December 1993)

\section{Introduction}

One of the outstanding problems in four-dimensional topology is to find the minimal genus of an oriented smoothly embedded surface representing a given homology class in a smooth four-manifold. For an arbitrary homology class in an arbitrary smooth manifold not even a conjectural lower bound is known. However, for the classes represented by smooth algebraic curves in (simply connected) algebraic surfaces, it is possible that the genus of the algebraic curve, given by the adjunction formula

$$
g(C)=1+\frac{1}{2}\left(C^{2}+C K\right)
$$

is the minimal genus. This is usually called the (generalized) Thom conjecture. It is mentioned in Kirby's problem list [11] as Problem 4.36.

There are a number of results on this question in the literature. They can be divided into two classes, those proved by classical topological methods, which apply to topologically locally flat embeddings, including the $G$-signature theorem, and those proved by methods of gauge theory, which apply to smooth embeddings only.

On the classical side, there is the result of Kervaire and Milnor[10], based on Rokhlin's theorem, which shows that certain homology classes are not represented by spheres. A major step forward was made by Rokhlin[20] and Hsiang and Szczarba [8] who introduced branched covers to study this problem for divisible homology classes. If the integral homology class represented by an embedded surface $\Sigma \subset X$ is divisible by $k$, then the corresponding cover of $X$ of order $k$ branched along $\Sigma$ is again a smooth 4-manifold, so there is an obvious inequality, simply from the existence of the covering, asserting that its second Betti number is at least the absolute value of its signature. In the case $k=2$, this gives

$$
g(\Sigma) \geqslant\left|\frac{1}{4} \Sigma^{2}-\sigma(X)\right|-b_{2}(X) .
$$

A better bound follows from the $G$-signature theorem [8], [20]. Again, we state it only for even homology classes:

$$
g(\Sigma) \geqslant\left|\frac{1}{4} \Sigma^{2}-\frac{1}{2} \sigma(X)\right|-\frac{1}{2} b_{2}(X)
$$

In the case of $C P^{2}$, if $\Sigma_{d}$ represents $d$ times a generator, and $d$ is even, the bound (2) gives $g\left(\Sigma_{d}\right) \geqslant \frac{1}{4} d^{2}-2$, and the $G$-signature theorem (3) gives $g\left(\Sigma_{d}\right) \geqslant \frac{1}{4} d^{2}-1$. The Thom conjecture asserts that $g\left(\Sigma_{d}\right) \geqslant \frac{1}{2} d^{2}-\frac{3}{2} d+1$.

\footnotetext{
* Partially supported by NSF grant DMS-91-07505.
} 
The applications of gauge theory to this problem were initially concerned with the representability of homology classes by spheres only. These are collected in the survey article by Lawson [16]. They usually relied on Donaldson's early theorems [2] on manifolds with $b_{2}^{+} \leqslant 2$. Next, Donaldson [3] used his polynomial invariants [4] for manifolds with $b_{2}^{+} \geqslant 3$ to prove that one cannot construct a counterexample to the Thom conjecture by doing surgery, i.e. killing a handle, on an ample curve. Substantial progress was made recently by Kronheimer and Mrowka[15] who developed gauge theory for singular connections with non-trivial holonomy around a loop linking an embedded surface $\Sigma$. They used this to prove that if $X$ has a non-trivial Donaldson invariant in the sense of [4], then

$$
g(\Sigma) \geqslant 1+\frac{1}{2} \Sigma^{2}
$$

except if $\Sigma$ is an inessential sphere or a sphere of self-intersection - 1 . In particular, this proves the Thom conjecture for $K 3$ surfaces. In other cases, however, it falls short of the bound (1). Even more recently, Kronheimer[14] announced a proof of the Thom conjecture for homology classes of positive self-intersection in certain surfaces of general type. The restriction to classes of positive self-intersection is reminiscent of the ampleness assumption in Donaldson's theorem [3].

In this paper we combine the classical method using branched covers with gauge theoretic arguments in two different ways to prove some results pertaining to the Thom conjecture for cases which are not addressed by the results of [3], [15] and [14].

First, using Donaldson's theorems [2] on spin manifolds with $b_{2}^{+} \leqslant 2$, we prove a lower bound on the genera of surfaces representing certain divisible classes in 4manifolds (Theorem 3.1). As a special case, we obtain:

THEOREM 1·1. Let $\Sigma_{d}$ be a smoothly embedded surface representing $d$ times the generator of $\mathrm{H}_{2}\left(\mathrm{CP}^{2}, \mathrm{Z}\right)$. If $d$ is even, $d>2$ and $\frac{1}{2} d$ is odd, then $g\left(\Sigma_{d}\right) \geqslant \frac{1}{4} d^{2}+1$.

This improves the best previously known bound by 2, and proves the Thom conjecture for degree 6 curves in $C P^{2}$. Lee and Wilczynski [17] have shown that the lower bounds proved in [8], [20] are realized by topologically locally flat surfaces, for all $d$ which are even or powers of an odd prime.

COROLLARY 1·2. For $d$ even, $d>2$ and $\frac{1}{2} d$ odd, the topologically locally flat surfaces of genus $g=\frac{1}{4} d^{2}-1$ representing dimes the generator of $H_{2}\left(\mathbf{C} P^{2}, Z\right)$ constructed in [17] are not smoothable.

There were earlier counterexamples to the topologically locally flat version of the Thom conjecture, due to Rudolph[21]; see also [22]. It follows from Theorem 1•1 that some of these are not smoothable, cf. Section 3.

In Section 3 we also give applications of Theorem $3 \cdot 1$ to other rational surfaces, including a proof of the Thom conjecture for the class $-2 K$ on any del Pezzo surface. As above, this shows that certain topologically locally flatly embedded surfaces are not smoothable.

Secondly, if a suitable branched cover has $b_{2}^{+} \geqslant 3$, then we prove in Theorem $5 \cdot 1$ that its Donaldson polynomials give obstructions to doing surgery on an embedded surface. This idea has already been used by Donaldson[3], and we generalize (and reprove) his result to show that under very general assumptions surgery cannot be done on complex curves to produce a counterexample to the Thom conjecture. 
TheOREM 5.2. Let $X$ be a simply connected smooth complex algebraic surface and $B \subset X$ a smooth algebraic curve. Then surgery cannot be done on $B$ if either

1. the self-intersection number $B^{2}$ is positive, or

2. [B] is divisible in $H_{2}(X, Z)$.

The second part is probably the more interesting one, because, unlike the results of [15] and [14], it applies to complex curves of non-positive self-intersection. Although the first part looks like an immediate generalization of Donaldson's result on ample curves, his proof does not generalize. By necessity, our proof is rather different. The main difference is that instead of using Donaldson's vanishing theorem for his polynomial invariants [4] of connected sums, we use the following theorem, proved in section 4, which says that certain homology classes can not be represented by spheres.

THeOREM 4:2. Let $X$ be a smooth closed oriented 4-manifold with $b_{1}(X)=0$ and $b_{2}^{+}(X)$ odd and $>1$. Suppose $X$ contains a smoothly embedded 2-sphere $S$ of zero self-intersection with $[S] \neq 0 \in \mathrm{H}_{2}(X, Q)$. Then all Donaldson invariants of $X$ vanish.

This theorem was first proved for the SU(2) Donaldson invariants of simply connected manifolds by Morgan, Mrowka and Ruberman (unpublished). That case is also covered by the results of [6] and by (4) and the proofs can probably be extended to the general case. However, our argument is much simpler, based, as it is, on a simple observation about the orientations of $\mathrm{SO}(3)$ moduli spaces [13].

To prove Theorem $\mathbf{5 \cdot 2}$ for curves which are not ample, we have to apply the vanishing theorem to a branched cover with non-trivial fundamental group. In this sense, we need the full strength of Theorem $4 \cdot 2$. See the final Remark in section 5 for a further comparison of Donaldson's proof in [3] with ours.

\section{Homology of branched covers}

Let $X$ be a closed oriented 4-manifold with $\pi_{1}(X)=1$, and $B \subset X$ a connected closed oriented surface. If $p$ is a prime such that $p^{r}$ divides $[B] \in H_{2}(X, Z)$, denote by $Y \rightarrow X$ the corresponding branched cover with covering group $Z_{p^{r}}$. The Betti numbers of $Y$ with coefficients in a field of characteristic zero and the signature of $Y$ were calculated in [8], [20] to be

$$
\begin{gathered}
b_{0}(Y)=b_{4}(Y)=1, b_{1}(Y)=b_{3}(Y)=0 \\
b_{2}(Y)=p^{r} b_{2}(X)+2\left(p^{r}-1\right) g(B) \\
\sigma(Y)=p^{r} \sigma(X)-\frac{p^{2 r}-1}{3 p^{r}} B^{2} .
\end{gathered}
$$

We have the following result concerning homology with integral coefficients:

Propositron 2.1. If $p^{r}$ is the maximal power of $p$ dividing $[B] \in H_{2}(X, \mathbf{Z})$, then the homology of $Y$ has no $p$-torsion.

Proof. Poincaré duality and the universal coefficient theorem imply that it is enough to show $H_{1}\left(Y, Z_{p}\right)=0$. 
Let $N \subset X$ be a tubular neighbourhood of the branch locus $B$, and $W=X-N$. If $d$ is the divisibility of $[B]$ in $H_{2}(X, \mathbf{Z})$, then $H_{1}(W, \mathbf{Z})=\mathbf{Z}_{d}$ [8], [20]. Let $V \rightarrow W$ be the unramified covering corresponding to the homomorphism

$$
\pi_{1}(W) \rightarrow H_{1}(W, \mathbf{Z}) \rightarrow \mathbf{Z}_{p^{r}}
$$

Adding the standard ramified cover $M \rightarrow N$ we obtain $Y=V \cup M$. The Mayer-Vietoris sequence for this decomposition gives

$$
H_{1}(\partial V) \rightarrow H_{1}(V) \oplus H_{1}(M) \rightarrow H_{1}(Y) \rightarrow 0,
$$

for homology with arbitrary coefficients. If we prove $H_{1}\left(V, Z_{p}\right)=0$, then, because $H_{1}(\partial V)=H_{1}(\partial M) \rightarrow H_{1}(M)$ is surjective, $H_{1}\left(Y, Z_{p}\right)=0$ as claimed.

Let $L=\pi_{1}(W)$ and $K=\pi_{1}(V)$. Then we have Diagram 1 of short exact sequences:

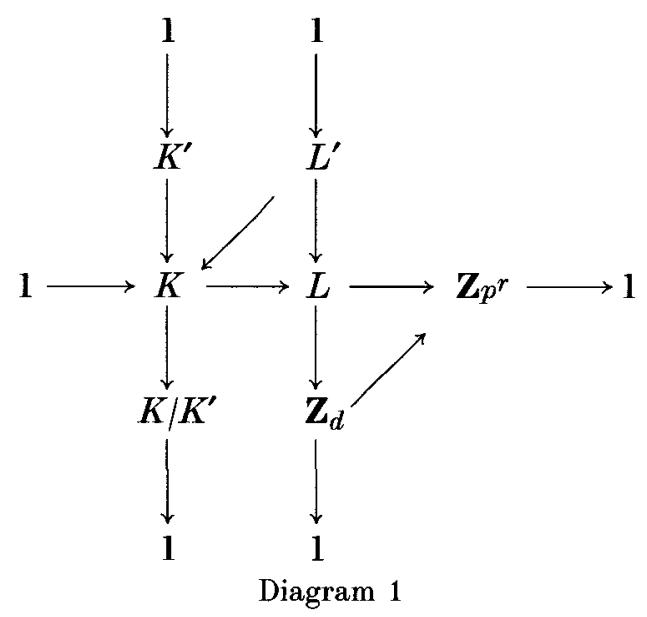

By Lemma $4 \cdot 1$ of [8] or section 3 of [20], $K / K^{\prime}=H_{1}(V)$ is a finite group $H$. We have to show that $p$ does not divide its order.

Lemma 2.2. Let $L$ and $K$ be as in Diagram 1 , with $K / K^{\prime}=H$ finite and $p^{r+1}$ not a divisor of $d$. Then $p$ does not divide the order of $H$.

The subgroup $K^{\prime} \subset L$ is normal. Dividing by $K^{\prime}$ and setting $G=L / K^{\prime}$, we obtain Diagram 2 of short exact sequence of groups :

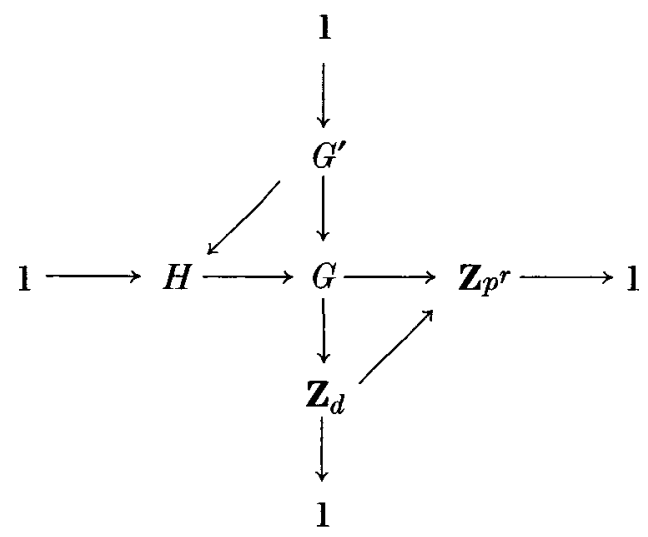

Diagram 2 
Note that $H$ is finite Abelian and contains $G^{\prime}$ as a subgroup. The claim is that $p$ does not divide $|H|$, which is equivalent to $p$ not dividing $\left|G^{\prime}\right|$. The factor group $V=G^{\prime} /\left\{x^{p} \mid x \in G^{\prime}\right\}$ is a vector space over $\mathbf{Z}_{p}$, and the claim is equivalent to $V=\mathbf{0}$.

Let $a \in G$ be such that its image in $\mathbf{Z}_{d}$ is a generator. The assignment $[x] \rightarrow\left[a x a^{-1}\right]$ gives a well-defined linear endomorphism $f$ of $V$. Since $a^{p^{r}} \in H$ and $H$ is Abelian, we have $f^{p^{r}}=I d_{V}$ and so $\left(I d_{V}-f\right)^{p^{r}}=I d_{V}-f^{p^{r}}=0$. Thus $I d_{V}-f$ is not surjective, unless $V=\mathbf{0}$.

The commutator $G^{\prime}$ is generated by elements of the form $a^{k} x a^{-k} x^{-1}$ with $x \in H$. Thus $V$ is generated by elements of the form $f^{k}(x)-x$. (We write the group operation in $V$ additively.) Putting $y=x+f(x)+\ldots+f^{k-1}(x)$, we have $f^{k}(x)-x=f(y)-y$. Thus $f-I d_{V}$ is surjective and we conclude $V=0$ as desired.

This completes the proof of the lemma and of the proposition.

Remark. It has been pointed out to us by P. Gilmer and O. Viro that the assumption of maximality in the Proposition can be dropped at the expense of using Smith theory [9]†. Proposition 2-1 suffices for all our applications, except the case of $S^{2} \times S^{2}$ in Corollary $3 \cdot 2$.

\section{A lower bound on the genera of embedded surfaces}

Combining the homology calculation of Section 2 with Donaldson's results in [2] we prove here as lower bound on the genera of embedded surfaces representing certain homology classes in a smooth simply connected 4-manifold.

Theorem 3-1. Let $X$ be a smooth oriented 4-manifold with $\pi_{1}(X)=1$, and $\Sigma \subset X a$ connected oriented smoothly embedded surface representing a non-zero homology class $[\Sigma] \in H_{2}(X, \mathbf{Z})$. Assume that $[\Sigma] \in H_{2}(X, \mathbf{Z})$ is divisible by $2^{r}$, that $\left(1 / 2^{r}\right)[\Sigma]$ is Poincaré dual to a lift of $w_{2}(X)$ to integral coefficients, and that

$$
\frac{2^{2 r}-1}{3 \cdot 2^{2 r}} \Sigma^{2} \neq \sigma(X)
$$

Then

$$
g(\Sigma) \geqslant\left|\frac{2^{r}+1}{3 \cdot 2^{r+1}} \Sigma^{2}-\frac{2^{r-1}}{2^{r}-1} \sigma(X)\right|-\frac{2^{r-1}}{2^{r}-1} b_{2}(X)+\frac{3}{2^{r}-1}
$$

Proof. Let $\pi: Y \rightarrow X$ be the $2^{r}$-fold cover branched along $\Sigma$. Then $w_{2}(Y)=$ $\pi^{*}\left(w_{2}(X)+P D\left(\left[\left(1 / 2^{r}\right) \Sigma\right]\right)=\pi^{*}(0)=0\right.$, where $P D(\alpha)$ denotes the Poincaré dual of $\alpha$. Thus $Y$ is a spin manifold. By Proposition $2 \cdot 1$ and the subsequent Remark, $H_{1}\left(Y, Z_{2}\right)=0$. Moreover, $\sigma(Y) \neq 0$ because $\left(\left(2^{2 r}-1\right) / 3.2^{2 r}\right) \Sigma^{2} \neq \sigma(X)$. Thus Donaldson's Theorems A, B and C in [2] apply to show that $\min \left\{b_{2}^{+}(Y), b_{2}^{-}(Y)\right\} \geqslant 3$. Substituting from the formulae (6) and (7) gives the claimed inequality.

If $b_{2}^{+}(X)$ is small, then this inequality improves the estimate of Hsiang and Szczarba [8] and Rokhlin [20] for the classes to which it applies. In particular, using $r=1$, it implies Theorem $1 \cdot 1$ in the Introduction.

$\dagger$ Note added in proof. We are grateful to J. Davis for showing us an argument to deduce this more general statement directly from Proposition $2 \cdot 1$. 
Another consequence is:

Corollary 3'2. Let $X$ be a rational surface with the class $-2 K_{X}$ represented by a smooth complex curve (where $K_{X}$ is the canonical divisor of $X$ ). Then the Thom conjecture holds for this class.

Proof. The surface $X$ is diffeomorphic to $\mathbf{C} P^{2} \# k \overline{\mathbf{C} P^{2}}$, or to $S^{2} \times S^{2}$. The genus of the complex curve representing $-2 K_{X}$ is given by the adjunction formula (1) to be $g\left(-2 K_{X}\right)=1+K_{X}^{2}=10-k$ in the first case and $g\left(-2 K_{X}\right)=1+K_{X}^{2}=9$ in the second. Using $r=1$ and substituting in (8) one sees that these values are the smallest possible.

Remark. This corollary proves the Thom conjecture for degree 6 curves in $\mathbf{C P}^{2}$ as does Theorem 1.1. It was previously proved for degrees 3 ([10], [8], [20]) and 4 (8], [20]). We can recover the result in the degree 4 case from Theorem 3.1 using $r=2$. The result for $d=3$ follows from that for $d=6$. Indeed, suppose there was a counterexample in degree 3 . This would be a sphere $S$. One could make a small perturbation $S^{\prime}$ of $S$ such that $S$ and $S^{\prime}$ have precisely 9 intersection points. Replacing the intersection points by handles, one would obtain a counterexample in degree 6 .

Remark. Rudolph [21] showed that for every $d \geqslant 6$ there is a topologically locally flat counterexample to the Thom conjecture. By Theorem 1.1, this is not smoothable for $d=6$. In [22], there is an example of a topologically locally flat embedding of a surface of genus 5 representing 5 times the generator of $H_{2}\left(C P^{2}, Z\right)$, which has geometric intersection number 5 with a complex line. This, too, cannot be smoothable, since by replacing the intersection points with handles, one would obtain an embedded surface of degree 6 and genus 9, contradicting Theorem 1·1.

The following corollary answers the question posed by L. Taylor in [11], Problem $4 \cdot 26$.

Corollary 3·3. Let $X=\mathbf{C} P^{2} \# 8 \overline{\mathbf{C} \bar{P}^{2}}$. The class $6 \gamma_{0}+2 \gamma_{1}+\ldots+2 \gamma_{8}$ in the canonical generators of $\mathrm{H}_{2}(X, \mathbf{Z})$ cannot be represented by a smoothly embedded sphere or torus.

Proof. Using $r=1$ again, the lower. bound from (8) gives $g \geqslant 2$ for this class.

Taylor (loc. cit.) notes that this class can be represented by a smoothly embedded surface of genus 2 .

Remark. Suciu [23] proved lower bounds on the number of transverse double points of immersed spheres representing certain homology classes in rational surfaces using Donaldson's Theorem A from [2]. Unlike our argument which applies gauge theory on a cover, Suciu applied Theorem A directly on $X$ or a suitable blowup or blowdown. By replacing double points with small handles, the lower bound we have proved on the genus of embedded surfaces gives a lower bound on the number of double points. In the cases to which our theorem applies, it improves Suciu's estimates. For example for immersed spheres in $\mathbf{C} P^{2}$ representing $d$ times the generator, with $d$ twice an odd number, the lower bound on the number of double points we obtain is one larger than the bound proved in [23].

Remark. It has been conjectured (cf. [5]) that every smooth simply connected spin 4-manifold $Y$ satisfies $8 b_{2}(Y) \geqslant 11 \sigma(Y)$. Donaldson's theorems that we have used in 
the proof of (8) can be thought of as special cases of this conjecture, generalized to manifolds with $H_{1}\left(Y, Z_{2}\right)=0$. If this general form of the ' $11 / 8$ ' conjecture is true, then under the same assumptions as Theorem $3 \cdot 1$ our argument above gives a much better inequality than $(8)$ :

$$
g(\Sigma) \geqslant \frac{11}{8}\left|\frac{2^{r}+1}{3 \cdot 2^{r+1}} \Sigma^{2}-\frac{2^{r-1}}{2^{r}-1} \sigma(X)\right|-\frac{2^{r-1}}{2^{r}-1} b_{2}(X) .
$$

For the special case of a surface $\Sigma_{d}$ in $\mathbf{C} P^{2}$, with $d$ even and $\frac{1}{2} d$ odd, one would have $g(\Sigma) \geqslant \frac{11}{32} d^{2}-\frac{19}{9}$. This would be a substantial improvement, but would still be weaker than the Thom conjecture, compare (1).

Note that the ' $11 / 8$ ' conjecture is true for connected sums of spin algebraic surfaces with $H_{1}\left(Y, Z_{2}\right)=0$, by Moishezon's argument given in [5] for the simply connected case.

\section{4. $S O(3)$-invariants and a vanishing theorem}

Throughout this section, $X$ denotes a smooth closed oriented 4-manifold with $b_{1}(X)=0$ and $b_{2}^{+}(X)$ odd and greater than 1 .

Donaldson [4] defined polynomial invariants for $X$ under the additional assumption that $X$ is simply connected. His definition works for $\mathrm{SU}(2)$-bundles with large enough second Chern class, and for all $\mathrm{SO}(3)$-bundles with non-zero second Stiefel-Whitney class $w_{2}$ for which the predicted dimension of the moduli space of anti-self-dual connections is non-negative. Donaldson's definition was extended in various ways, to remove both the restriction on the second Chern class of SU(2)bundles [6] and the assumption on the fundamental group [18]. It was pointed out in [13] that if $b_{1}(X)=0$, then Donaldson's original definition of polynomial invariants goes through if one uses bundles $P \rightarrow X$ with $w_{2}(P)$ the mod 2 reduction of some integral class and not a pullback from $H^{2}\left(K\left(\pi_{1}(X), 1\right), Z_{2}\right)$. This is because the moduli spaces of anti-self-dual connections have a fundamental homology class, due to the fact that no flat connections arise in their compactifications.

As a point of notation, we will refer to the invariants defined using these special $\mathrm{SO}(3)$-bundles as $\mathrm{SO}(3)$-invariants. The term Donaldson invariants will be used more generally to denote all of the above-mentioned invariants.

Let $P \rightarrow X$ be an $\mathrm{SO}(3)$-bundle with $w_{2}(P)$ not a pullback from $H^{2}\left(K\left(\pi_{1}(X), 1\right), Z_{2}\right)$. Denote by $2 d$ the dimension of the moduli space of anti-self-dual connections on $P$, with respect to a generic Riemannian metric. To fix the orientation of the moduli space, one needs to choose (an equivalence class of) a lift $c$ of $w_{2}(P)$ to integral cohomology. We assume that such a lift exists. If $\alpha \in H_{2}(X, Z)$, then according to [13] the class $2 \mu(\alpha)$ is an integral class on any family of connections. (Here we use Donaldson's definition $\mu(\alpha)=-\frac{1}{4} p_{1}(\mathbf{P}) / \alpha$, where $\mathbf{P}$ is a universal SO(3)-bundle.) We evaluate $d$-fold cup products of the classes $2 \mu(\alpha)$ on the fundamental class of the moduli space. The resulting invariant is an integral polynomial of degree $d$ and will be denoted $\Phi_{d, c}^{X}$.

Proposition 4.1 ([13]). Consider an $\mathrm{SO}(3)$-bundle $P \rightarrow X \# \overline{\mathbf{C} P^{2}}$ with $w_{2}(P)$ non-trivial on $\mathrm{H}_{2}\left(\overline{\mathrm{C} P^{2}}\right)$. The corresponding $\mathrm{SO}(3)$-invariant $\Phi_{a+1, c-h}^{X * \overline{\mathrm{C} P^{2}}}$ is an odd polynomial in the generator $h \in H^{2}\left(\overline{\mathbf{C} P^{2}}, \mathbf{Z}\right)$. Moreover,

$$
\Phi_{d+1, c-h}^{X * \overline{C P^{2}}}=2 h \Phi_{d, c}^{X}+O\left(h^{3}\right) .
$$


Proof. If $w_{2}(P)$ is non-trivial on $H_{2}\left(\overline{\mathbf{C} P^{2}}\right)$, then it is not a pullback from $K\left(\pi_{1}\left(X \# \overline{\mathbf{C} P^{2}}\right), 1\right)$. Thus, if it has a lift to integral cohomology, there are associated $\mathrm{SO}(3)$-invariants.

The reflection in $h$ on the cohomology of $X \# \overline{\mathbf{C} P^{2}}$ is realized by a selfdiffeomorphism of the manifold, which changes the orientation of the moduli space. This implies that the polynomial is odd in $h$. See [13] for details.

The precise formula for the leading order term is proved by degenerating the metric on the connected sum to shrink the connecting tube. In the limit, anti-selfdual connections decompose into anti-self-dual connections on the two summands. This splitting is restricted by the decomposition of $w_{2}(P)$. For the leading term one only has to consider the unique reducible connection with $p_{1}=-1$ on $\overline{\mathbf{C} P^{2}}$. Gluing this to connections on the bundle $P$ restricted to $X$, the moduli space over $X \# \overline{\mathbf{C} P^{2}}$ is seen to be an $S^{2}$-bundle over a moduli space of connections on $X$. The $S^{2}$ is the gluing parameter $\mathrm{SO}(3)$ divided by the stabilizer of the reducible connection. It is the evaluation of the class $2 \mu(P D(h))$ on the fibre $S^{2}$ which produces the factor $2 h$ in the formula. If $\Phi_{a, c}^{X}$ was well-defined by any one of the methods in [4], [6], [18], then it appears here as the contribution from the base of the $S^{2}$-fibration. If not, the term appearing can be taken as a definition of the appropriate invariant of $X$. Again, details of such arguments have appeared in [13] and [6].

Remark. If one uses the classes $\mu(\alpha)$ instead of $2 \mu(\alpha)$, as is done in [18], then the formula for the leading order term does not have a factor of 2 in it. Thus, the formulae in [18] have unnecessary powers of 2 in them.

As an application of this proposition we prove a general vanishing theorem for Donaldson invariants.

Theorem 4.2. Suppose $X$ contains a smoothly embedded 2-sphere $S$ of zero self-intersection with $[S] \neq 0 \in H_{2}(X, Q)$. Then all Donaldson invariants of $X$ vanish.

Proof. As above, if $X$ has some non-trivial Donaldson invariant $\Phi_{d, e}^{X}$, then $X \# \overline{\mathbf{C} P^{2}}$ has a non-trivial $\mathrm{SO}(3)$-invariant $\Phi_{a+1, c-h}^{X * \overline{\mathrm{CP}}}$ defined using a bundle $P$ with $w_{2}(P)=$ $c-h(\bmod 2)$ and therefore non-trivial on $H_{2}\left(\overline{\mathbf{C} P^{2}}\right)$.

Tubing together $2 n$ parallel copies of $S$ and one copy of the sphere $E$ of self-intersection -1 in $\overline{\mathbf{C} P^{2}}$, we find that for all $n$ the class $\alpha_{n}=2 n[S]+[E]$ is represented by a smoothly embedded 2 -sphere with self-intersection number -1 on which $w_{2}(P)$ evaluates non-trivially. By the Proposition, the Poincaré dual of the class $\alpha_{n}$ divides $\Phi_{d+1, c-h}^{X \# \overline{C P^{2}}}$.

But the classes $\alpha_{n}$ are all distinct because [S] is non-trivial in rational homology. Thus $\Phi_{a+1, c-h}^{X * \overline{C P^{2}}}$ has infinitely many linear factors. As it has finite degree, it must vanish identically. A fortiori, $\Phi_{a, c}^{X}$ vanishes identically.

Remark. This argument applies not only to invariants for manifolds with $b_{2}^{+} \geqslant 3$. It applies just as well to the invariant $\phi$ for manifolds with $b_{2}^{+}=1$ introduced in [12], because that invariant and the corresponding one on the connected sum $X \# \overline{\mathbf{C} P^{2}}$ are absolute invariants, i.e. they do not depend on a chamber structure [13]. Thus, the argument shows that a manifold for which $\phi$ is nonzero, e.g. the Barlow surface or the classical Godeaux surface [12], does not contain any smoothly embedded essential sphere of zero selfintersection. 


\section{Surgery on embedded surfaces}

Let $X$ be a smooth closed oriented 4-manifold with $\pi_{1}(X)=1$, and $B \subset X$ a smoothly embedded closed oriented surface.

We say that surgery can be done on $B$ if there is a simple closed curve $\delta \subset B$ with $[\delta] \neq 0 \in H_{1}(B)$ which bounds an embedded 2-disc $D \subset X-B$ in such a way that the section of the normal bundle to $D$ defined by $B$ over $\delta$ extends to the interior of $D$ as a nowhere vanishing section. Then two parallel copies of $D$ can be attached to $B$ cut along parallel copies of $\delta$ to lower the genus of $B$ without changing its homology class.

For any $n \in \mathbf{Z}$ the class $n[B]$ can be represented by a smoothly embedded surface $B_{n}$ contained in a tubular neighbourhood of $B$. For every prime $p$ let $p^{r(p, n)}$ be the maximal power of $p$ which divides $\left[B_{n}\right]$ in $H_{2}(X, Z)$ and $Y_{n, p} \rightarrow X$ the corresponding cover of order $p^{r(p, n)}$ branched along $B_{n}$.

THEOREM 5-1. If for some $n$ and some $p$ with $r(p, n)>0$ the covering $Y_{n, p}$ has a non-trivial Donaldson invariant, then surgery cannot be done on $B$.

Proof. Note that $b_{1}\left(Y_{n, p}\right)=0$ by (5), so that the arguments of the previous section can be applied to $Y_{n, p}$.

Suppose $Y_{n, p}$ has a non-trivial Donaldson invariant. If $B$ can be surgered along some oriented curve $\delta$, then $\delta$ can be isotoped so that it lifts to $n$ disjoint copies in $B_{n}$, each of which can be used to surger $B_{n}$, cf. [3] p. 95. Thus, it suffices to prove that surgery can not be done on $B_{n}$.

We may assume $n=1$ and set $Y=Y_{1, p}$ and $k=p^{r(p, 1)}$. The surgering $\operatorname{disc} D$ for $B$ lifts to $k$ copies with disjoint interiors in $Y$. Gluing together any two of these with opposite orientations along their common boundary we find a smoothly embedded 2sphere with zero self-intersection number.

If one of these 2-spheres is non-trivial in rational homology, then the vanishing Theorem $4 \cdot 2$ contradicts the assumption that $Y$ has a non-trivial Donaldson invariant. This would complete the proof.

Let $\gamma \subset B$ be an oriented simple closed curve intersecting the surgering curve $\delta$ transversely and precisely once. Denote the intersection point by $P$. In $X$ the curve $\gamma$ bounds an oriented smoothly embedded surface $C$ which can be chosen in such a way that its interior is disjoint from $B$, its interior intersects the interior of $D$ transversely and $T_{P} D \oplus T_{P} C=T_{P} X$. Let $a \in T_{P} D$ be an inward pointing normal vector to the boundary of $D$ and $b \in T_{P} C$ be an inward pointing normal vector to the boundary of $C$. Then $a$ and $b$ span a disc normal to $B$ at $P$. The above choices can be made in such a way that $a \wedge b$ agrees with the orientation of the normal disc defined by the orientations of $X$ and $B$.

The branched cover of the normal disc is an oriented disc in $Y$, and the preimages of the intersection of $D$ with the normal disc can be labelled $D_{0}, \ldots, D_{k-1}$ by going around the boundary of this oriented disc in the positive direction. See Figure 1. The preimage $C^{\prime}$ of $C$ in $Y$ is a $Z_{p}$-manifold and in the normal disc to $B$ there is one sheet of $C^{\prime}$ between $D_{i}$ and $D_{i+1}$, for each $i$. See Figure 1 again.

Now $C^{\prime}$ defines a $Z_{p}$-cycle whose intersection number with the sphere $S=D_{1}-D_{0}$ is 1 . To see this, observe that the intersection points of $C^{\prime}$ with the interiors of $D_{1}$ and $D_{0}$ cancel each other. There is one other intersection point, the preimage of $P$ in the 

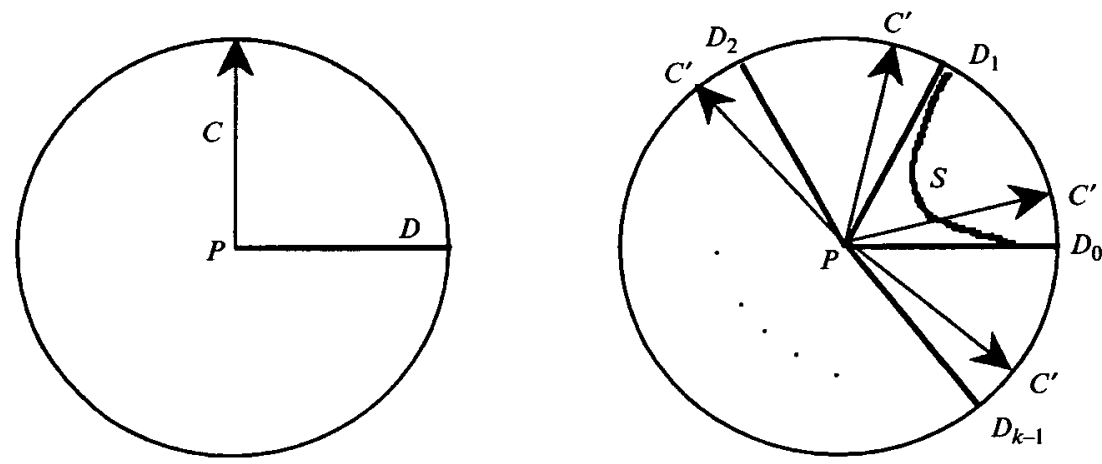

Figure 1: The normal disc to $B$ at $P$ in $X$ and in the $k$-fold cover

singular set of $C^{\prime}$. Perturbing $S$ to make this intersection transverse gives intersection number 1.

Thus [S] is non-trivial in $H_{2}\left(Y, Z_{p}\right)$. By Proposition $2 \cdot 1$ the integral homology of $Y$ is free of $p$-torsion. We conclude that $[S] \neq 0 \in H_{2}(Y, Q)$.

This concludes the proof of the Theorem.

Remark. The assumption about the maximality of the prime power $p^{r(p, n)}$ in this theorem is there to ensure that we can apply Proposition $2 \cdot 1$ to conclude that the branched cover has no $p$-torsion.

Instead of beginning with $X$ and constructing some covering $Y$, one can sometimes start from $Y$ and apply a variant of this argument, as in the following example.

Example. Let $Y$ be a smooth complex algebraic surface with $H_{1}\left(Y, Z_{2}\right)=0$ and with positive geometric genus. If $\sigma: Y \rightarrow Y$ is an anti-holomorphic involution with nonempty connected orientable fixed locus $B$, then $B$ is a smoothly embedded surface in the smooth manifold $X=Y / \sigma$ and our argument shows that surgery cannot be done on $B$.

There is an alternative way to prove this, based on the lower bound (4) proved by Kronheimer and Mrowka[15]. In fact, the fixed locus $B$ satisfies the equality $2 g(B)-2=B^{2}$ in $Y$, so that it has minimal genus in its homology class. If $B$ could be surgered in $X$, then it could also be surgered in $Y$, contradicting the minimality.

Our main application of Theorem $5 \cdot 1$ is to show that the naive approach to constructing a counterexample to the Thom conjecture fails in almost all cases.

TheOREM 5·2. Let $X$ be a simply connected smooth complex algebraic surface and $B \subset X$ a smooth algebraic curve. Then surgery cannot be done on $B$ if either

1. the self-intersection number $B^{2}$ is positive, or

2. $[B]$ is divisible in $H_{2}(X, Z)$.

Proof. We reduce the proof of the first case to that of the second one as follows. By a theorem of Hartshorne (Theorem 4.2 in [7]), the linear system $|n B|$ is free of base points if $B^{2}>0$ and $n$ is sufficiently large. Thus, using Bertini's theorem, we can choose a surface $B_{n}$ as in the proof of Theorem 5.1 to be a smooth algebraic curve supported near $B$. If the original curve can be surgered, then so can the new one, as in the proof of Theorem 5.1. Therefore, we may assume that we are in the second case.

If $[B]$ is divisible in integral homology we apply Theorem $5 \cdot 1$ in the following way. Let $p^{r}>1$ be the maximal power of some prime $p$ which divides $[B]$ in $H_{2}(X, Z)$. 
Consider the branched cover $Y \rightarrow X$ of order $p^{r}$ whose branch locus is $B$. Then $Y$ is a regular algebraic surface and so has a non-trivial Donaldson invariant as soon as $p_{g}(Y)>0$. This condition is trivially satisfied if $p_{g}(X)>0$. In general, we have $p_{g}(Y) \geqslant h^{0}\left(K_{X}+\left(\left(p^{r}-1\right) / p^{r}\right) B\right)$. Using Serre duality, $h^{0}\left(K_{X}+\left(\left(p^{r}-1\right) / p^{r}\right) B\right)$ can be estimated from below by the holomorphic Euler characteristic, which is computed from the Riemann-Roch theorem:

$$
p_{g}(Y) \geqslant \chi\left(K_{X}+\frac{p^{r}-1}{p^{r}} B\right)=1+p_{g}(X)+\frac{p^{r}-1}{2 p^{r}} K_{X} B+\frac{\left(p^{r}-1\right)^{2}}{2 p^{2 r}} B^{2} .
$$

The only case when there is anything to prove is when $g(B)>0$, which, by the adjunction formula (1), is equivalent to $K_{X} B+B^{2} \geqslant 0$. Using this, we find

$$
p_{g}(Y) \geqslant 1+p_{g}(X)-\frac{p^{r}-1}{2 p^{2 r}} B^{2} .
$$

This shows that $p_{g}(Y)>0$, unless possibly if $B^{2}>0$. In this last case, by replacing $B$ with a large enough multiple $B_{n}$ with $p$ not dividing $n$, we may assume that the linear system $\left|\left(\left(p^{r}-1\right) / p^{r}\right) B\right|$ contains a smooth curve of positive genus. Thus, using (11) again,

$$
p_{g}(Y) \geqslant 1+p_{g}(X)+\frac{p^{r}-1}{2 p^{r}} K_{X} B+\frac{\left(p^{r}-1\right)^{2}}{2 p^{2 r}} B^{2}=p_{g}(X)+g\left(\frac{p^{r}-1}{p^{r}} B\right)>0 .
$$

This completes the proof of the theorem.

The following example shows that the argument replacing $B$ by a multiple $B_{n}$ cannot be avoided, even when one assumes that $[B]$ is divisible.

Example. Let $X$ be the blowup of $\mathrm{CP}^{2}$ in one point, and $B$ a smooth curve in the linear system $|4 H-2 E|$, where $H$ is the pullback of a hyperplane section of the plane and $E$ is the exceptional curve. Then $g(B)=2$ and the divisibility of $[B]$ in $H_{2}(X, Z)$ is 2. The corresponding double cover $Y$ has $p_{g}(Y)=p_{g}(X)+h^{0}\left(K_{X}+\frac{1}{2} B\right)=0$. In fact, $Y$ is a rational surface diffeomorphic to $\mathbf{C} P^{2} \# 7 \overline{\mathbf{C} P^{2}}$.

Remark. As mentioned in the introduction, Theorem 5.2 is a generalization of Donaldson's result [3] for ample curves. In his proof, ampleness is used in several different ways.

It is used to replace the curve $B$ by a multiple, in order to make its homology class divisible and to make the branched cover have $p_{g}>0$. If $B^{2} \leqslant 0$, then the estimate (12) shows that the geometric genus of the cover is positive, so there is no need to pass to a multiple as we assume that $[B]$ is divisible. If $B^{2}>0$, then we use Hartshorne's theorem to pass to a multiple.

In [3] ampleness is also used via the Lefschetz hyperplane theorem to conclude that $\pi_{1}(X-B)=\mathbf{Z}_{a}$. This implies on the one hand that the branched cover is simply connected, and, on the other, that $B$ is isotopic to the surgered surface $\Sigma$ with a small handle attached. We deal with the first point by doing gauge theory on manifolds with $b_{1}=0$ as in [13]. The question of the isotopy is not so easily dealt with. For an arbitrary smooth algebraic curve in an algebraic surface the fundamental group of the complement is not understood, and can be very large. It is this problem which forced us to abandon the line of proof of [3]. When the isotopy can be done, as in [3], 
then a double branched cover splits off a copy of $S^{2} \times S^{2}$ and one can apply Donaldson's vanishing theorem for connected sums [4]. When the isotopy cannot be done, then the surgering dise still produces essential embedded spheres of zero self-intersection in the cover, but there is no obvious connected sum decomposition. This is why we use Theorem $4 \cdot 2$ instead of Donaldson's vanishing theorem.

It seems an interesting problem to understand the fundamental groups of complements of (smooth) curves in algebraic surfaces. Only in the case of curves of positive self-intersection (with some additional hypotheses) have we found results in the literature, ef. [1], [19].

It is a pleasure to thank Mikio Furuta and Peter Kronheimer for helpful discussions.

\section{REFERENCES}

[1] F. Catanese. On the moduli spaces of surfaces of general type, J. Differential Geometry 19 (1984), 483-515.

[2] S. K. Donaldson. The orientation of Yang-Mills moduli spaces and 4-manifold topology. $J$. Differential Geometry 26 (1987), 397-428.

[3] S. K. Donaldoson. Complex curves and surgery. Publ. Math. IHES 68 (1989), 91-97.

[4] S. K. Donaldson. Polynomial invariants for smooth four-manifolds. Topology 29 (1990), 257-315.

[5] R. Friedman and J. W. Morgan. Algebraic surfaces and 4-manifolds: some conjectures and speculations. Bull. (New Ser.) Am. Math. Soc. 18 (1988), 1-19.

[6] R. Friedman and J. W. Morgan. Smooth 4-manifolds and complex surfaces (Springer-Verlag, 1994).

[7] R. Hartshorne. Ample subvarieties of algebraic varieties. Lecture Notes in Mathematics 156 (Springer-Verlag, 1970).

[8] W. C. Hstang and R. H. Szczarba. On embedding surfaces in four-manifolds. Proc. Symp. Pure Math. 22 (1971), 97-103.

[9] V.S. ItenkeRg. Medium-dimension homologies of a submanifold of codimension two. Func. Analysis Appl. 8 (1974), 121-126.

[10] M. A. Kervaire and J. W. Milnor. On 2-spheres in 4-manifolds. Proc. Nat. Acad. Sci. U.S.A. 47 (1961), 1651-1657.

[11] R. Kirby. Problems in low-dimensional topology. Proc. Symp. Pure Math. 32 (1978), 273-322.

[12] D. Kotschick. On manifolds homeomorphic to $C P^{2} \# 8 \overline{\mathrm{C} P^{2}}$. Invent. math. 95 (1989), 591-600.

[13] D. Kotschick. SO(3)-invariants for 4-manifolds with $b_{2}^{+}=1$. Proc. London Math. Soc. 63 (1991), 426-448.

[14] P. B. Kronheimer. The genus-minimizing property of algebraic curves. Bull. Amer. Math. Soc. (New Series) 29 (1993), 63-69.

[15] P. B. Kronhemmer and T. S. Mrowka. Gauge theory for embedded surfaces I, II. Topology (to appear).

[16] T. LAwSON. Smooth embeddings of 2-spheres in 4-manifolds. Expo. Math. 10 (1992), 289-309.

[17] R. LEE and D. WILCZYNSKI, (in preparation).

[18] J. W. Morgan and T. S. Mrowka. A note on Donaldson's polynomial invariants. Int. Math. Research Notices 10 (1992), 223-230.

[19] M. V. Nort. Zariski's conjecture and related problems. Ann. Sci. École Norm. Sup. 16 (1983), 305-344.

[20] V. A. Rokhlin. Two-dimensional submanifolds of four-dimensional manifolds. Func. Analysis Appl. 6 (1971), 39-48.

[21] L. RuDOLPH. Some topologically locally-flat surfaces in the complex projective plane. Comment. Math. Helv. 59 (1984), 592-599.

[22] L. Rudolph. Quasipositivity as an obstruction to sliceness. Bull. Amer. Math. Soc. (New Series) 29 (1993), 51-59.

[23] A. I. Suclu. Immersed spheres in $C P^{2}$ and $S^{2} \times S^{2}$. Math. Zeitschrift 196 (1987), 51-57. 